\title{
Insight Into Osseointegration of Nanohydroxyapatite/Polyamide 66 Based on the Radiolucent Gap: Comparison With Polyether-Ether-Ketone
}

\author{
Jianxiao $\mathrm{Li}^{1}$, Haitao Peng ${ }^{2}$, Zhiyu Chen ${ }^{1}$, Chenbo $\mathrm{Hu}^{1}$, Tao $\mathrm{He}^{1}$, Hong $\mathrm{Li}^{2}$ and \\ Zhengxue Quan ${ }^{1 *}$ \\ ${ }^{1}$ Department of Orthopedics, The First Affiliated Hospital of Chongqing Medical University, Chongqing, China, ${ }^{2}$ College of \\ Physics, Sichuan University, Chengdu, China
}

Spinal fusion cages have been used in spinal fusion surgery for over 20 years. Polyether-etherketone (PEEK) cages are one of the most widely used materials. However, an increasing number of clinical and preclinical studies have shown that as a bioinert material the PEEK cage causes implant failure owing to limited osseointegration. The most common complication is a radiolucent zone at the bone-implant interface. Nanohydroxyapatite/polyamide 66 (n-HA/PA66) is a bioactive composite with sufficient load-bearing properties and good osseointegration abilities. However,

OPEN ACCESS

Edited by:

Derek H Rosenzweig, McGill University, Canada

Reviewed by: Rahul Gawri, McGill University, Canada Luciano Vidal,

Ecole Centrale de Nantes, France

*Correspondence:

Zhengxue Quan quanzx18@126.com

Specialty section: This article was submitted to Biomaterials,

a section of the journa

Frontiers in Materials

Received: 09 March 2021 Accepted: 12 May 2021

Published: 04 June 2021

Citation:

Li J, Peng H, Chen Z, Hu C, He T, LiH and Quan Z (2021) Insight Into Osseointegration of Nanohydroxyapatite/Polyamide 66 Based on the Radiolucent Gap: Comparison With Polyether-Ether-

Ketone.

Front. Mater. 8:678550. doi: $10.3389 /$ fmats.2021.678550 in the early stage after surgery, a radiolucent gap can also be observed at the margin of the boneimplant interface. To better assess osseointegration performance as a fusion cage and compare the radiolucent gaps between the two materials, PEEK and n-HA/PA66, implants were prepared and implanted into the femoral condyles of adult New Zealand white rabbits to create a line-to-line bone-implant interface model. The interfaces were systematically investigated using X-ray radiography, histological analysis, scanning electron microscopy (SEM), elemental mapping analysis, micro-computed tomography evaluation, and push-out tests at 4, 8, 12, 24, and 52 weeks. Analysis of X-ray films and histological sections indicated a radiolucent gap around the margin of n-HA/PA66 in the early weeks after implantation (4-8 weeks). The gap narrowed and decreased gradually at 24-52 weeks. Histological analysis and SEM suggested that the formed bone could integrate and adhere in some regions of the implant surface. In addition, a better bonelike apatite layer was formed between the bone and the n-HAPA66 implant interface than with the PEEK implant. Push-out tests conducted at 24 and 52 weeks to evaluate integrated strength showed that the n-HA/PA66 implants have better bonding strength and sufficient stability, whereas PEEK implants possess poor integrated strength. Therefore, the n-HAVA66 composite exhibits good osseointegration properties and an improved integrated bone-implant interface.

Keywords: osseointegration, bioactive material, bone-implant interface, spinal fusion, hydroxyapatite/polyamide 66, polyetheretherketone

\section{INTRODUCTION}

Interbody spinal fusion using interbody fusion cages has many advantages for the treatment of spinal disorders. Fusion cages have been clinically used routinely for more than 20 years with a variety of implant materials and different designs (Albrektsson and Jacobsson, 1987; Bagby, 1988; Cloward, 2007). Most currently used fusion cages are constructed from polyether-ether-ketone (PEEK) 


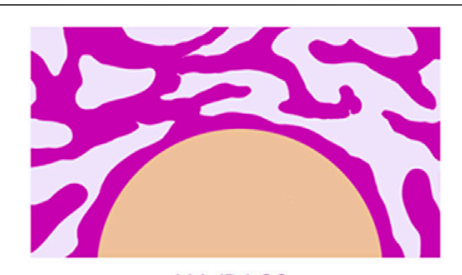

n-HA/PA66

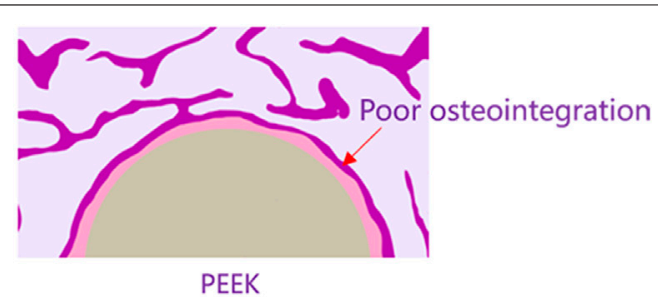

PEEK

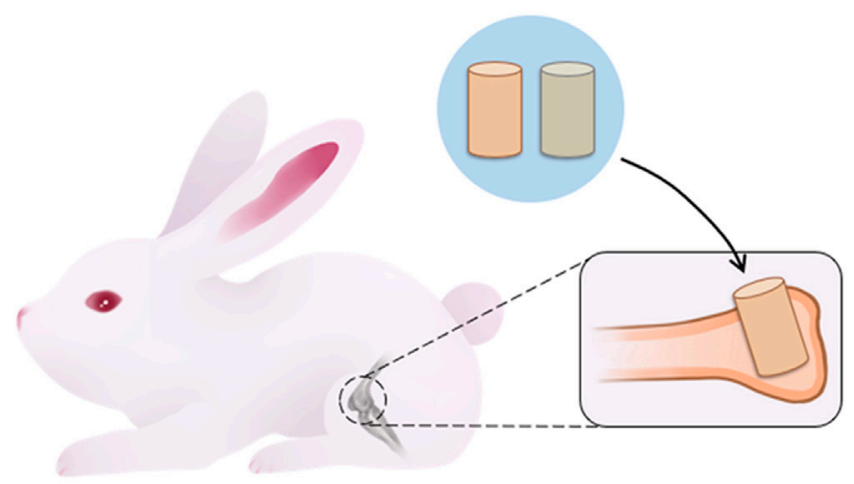

animal experiment

GRAPHICAL ABSTRACT | schematic diagram.

materials. Owing to its good biocompatibility, a cortical bone with a similar elastic modulus and mechanical characteristics has now been widely used in spinal fusion or non-fusion, trauma, and neurosurgical and cranio-maxillofacial surgeries for more than 15 years (Toth et al., 2006; Kurtz and Devine, 2007). However, bone does not bond to PEEK, a potential complication of PEEK is that its limited osseointegration with the surrounding bone results in a "PEEK-halo" effect (Phan et al., 2016). This "PEEK-halo" denotes a "radiolucent zone" that can be clearly seen on X-ray radiographs at the bone-implant interface, which may inhibit a successful fusion in the spinal interbody space. Radiolucent zones around the implant may be an indication of weak osseointegration, and fibrous encapsulation may be formed at the PEEK-bone interface, potentially leading to clinical failure with long-term implantation in the body (Noiset et al., 1999; Wu et al., 2013; Walsh et al., 2015).

Weak osteointegration affects the long-term stability of the interbody cages (Yuan et al., 2018). Much work has been conducted to improve the osteointegration property of interbody cages. Several interbody cages now have additional surface coating to enhance the bone-cage interface. Achieving long-term clinical success of interbody cage implants remains a significant and multifaceted challenge (Torstrick et al., 2018). Understanding the osseointegration of the cage materials will help the selecting and designing of new interbody cages materials in the future. The nanohydroxyapatite/polyamide 66 (n-HA/ PA66) and PEEK are two materials currently utilized in interbody cages. The n-HA/PA66 composite is a bioactive material with the ability to promote new bone formation and osteogenesis. However, in our previous studies, we discovered the presence of a "radiolucent gap" between the implant and the adjacent endplate bone in the radiographic images with this material also (Zhang et al., 2016). We evaluated: 1) whether the "radiolucent gap" is the same as the "Peek-halo" and 2) if n-HA/P66 exhibits an improved bone-implant interface compared to that of PEEK.

In the present study, to better identify the bone-implant interface and assess the long-term osseointegration effects between the n-HA/P66 and PEEK, we used bone defects in rabbits to create a line-to-line fit bone-implant interface model previously reported by the literature (Bertollo et al., 2011). $\mathrm{X}$-ray radiography and histology were used to observe the bone-implant interface. Micro-computed tomography (microCT) was used to evaluate new bone formation around the implant. Additionally, to evaluate the microstructure and dynamic change in chemical elements at the bone-implant interface, scanning electron microscopy (SEM) and energy dispersive X-ray spectroscopy (EDX) were performed at 4, $8,12,24$, and 52 weeks. Finally, to evaluate the integrated strength of implants and bone, the push-out test was applied at 24 and 52 weeks.

\section{MATERIALS AND METHODS}

\section{Sample Preparation}

The $\mathrm{n}-\mathrm{HA} / \mathrm{P} 66$ rods $(\varphi=6 \mathrm{~mm}, \mathrm{~L}=10 \mathrm{~mm})$ were provided by Sichuan Guona Technology Co., Ltd. (Chengdu, China) and had an HA content of approximately $40 \%$. The PEEK composite was provided by Shandong WEGO Orthopedic Device Co., Ltd. 

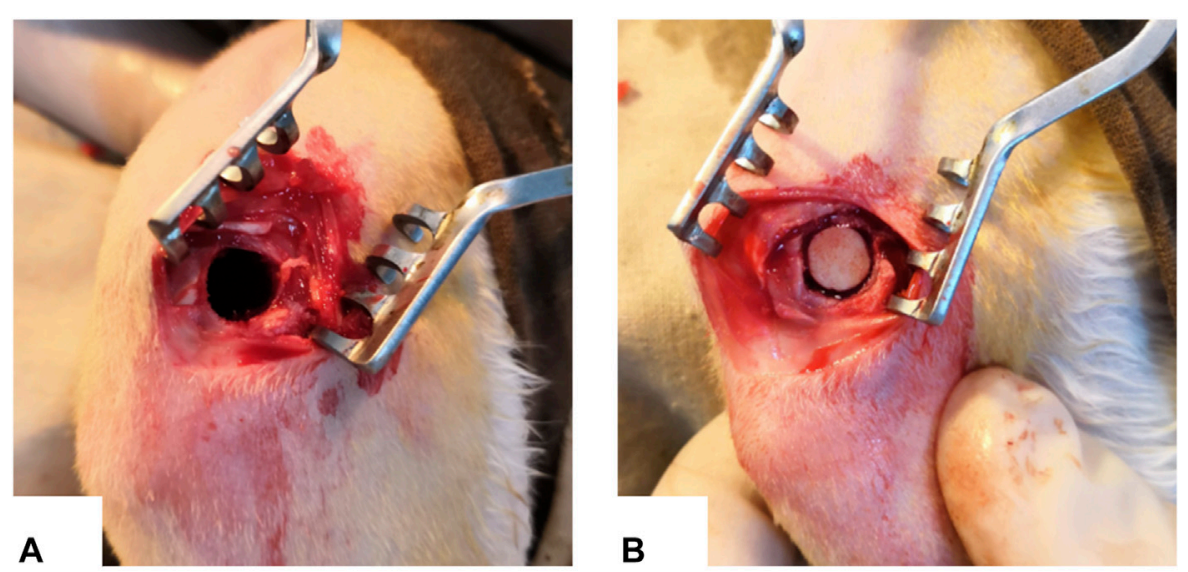

FIGURE 1 | (A) A line-to-line bone-implant interface model was constructed in the femoral condyle of New Zealand white rabbits using a 6 mm diameter defect. (B) An implant $6 \mathrm{~mm}$ in diameter was implanted into the defect region.

(Weihai, China). Commercially available PEEK rods $(\varphi=6 \mathrm{~mm}$, $\mathrm{L}=10 \mathrm{~mm}$ ) were used in this study. Prior to surgical implantation, the samples were sterilized by $\gamma$-ray irradiation at a dose of $25 \mathrm{kGy}$.

\section{Animal Model and Implantation Surgery}

The animal experiments were approved by the Ethical Committee of The First Affiliated Hospital of Chongqing Medical University. Thirty male New Zealand white rabbits (12 weeks old, body weight $2.8-3.0 \mathrm{~kg}$ ) were randomly divided into two groups. All animal experiments were performed following the "Guidance Suggestions for the Care and Use of Laboratory Animals" of the National Science and Technology Committee of the People's Republic of China, outlined in order No. 2, 2006. Surgeries were performed under general anesthesia by intravenous injection $(1.0 \mathrm{ml} / \mathrm{kg})$ with a $3 \%$ pentobarbital sodium solution (Sigma-Aldrich Co.) under sterile conditions. After proper preparation, a defect $(\varphi=$ $6 \mathrm{~mm}, \mathrm{~L}=10 \mathrm{~mm}$ ) was drilled through the femoral condyle using $4 \mathrm{~mm}$ diameter and $6 \mathrm{~mm}$ diameter sterile drills (Figure 1A). An n-HA/PA66 implant was implanted into the condyle of one femur, and a PEEK implant was implanted into the contralateral femoral condyle as the control (Figure 1B). The wounds were sutured in layers. Postoperatively, gentamicin $(5 \mathrm{mg} / \mathrm{kg})$ and penicillin $(50 \mathrm{kU} / \mathrm{kg})$ were administered intramuscularly for 3 days. After the operation, all animals were allowed to bear full weight and received a normal diet. At 4, 8, 12, 24, and 52 weeks after surgery, the animals were sacrificed via celiac injection of an excessive amount of pentobarbital sodium. Implants were harvested from the surrounding tissues and fixed in $4 \%$ formaldehyde at $4^{\circ} \mathrm{C}$ for 1 week.

\section{X-Ray Radiography and Micro-CT}

$\mathrm{X}$-ray radiographs were taken at $4,8,12,24$, and 52 weeks after implantation to investigate the interface between the material and bone tissue. The retrieved specimens at each time point $(4,8,12$,
24, and 52 weeks, $n=3$ ) were examined using micro-CT (VivaCT80, Scanco Medical AG, Bassersdorf, Switzerland; pixel size: $30 \mu \mathrm{m}$, scan voltage: $70 \mathrm{kV}$, scan current: $200 \mu \mathrm{A}$, integral time: $300 \mathrm{~ms}$ ).

A global threshold was utilized to segment the newly formed bone from each implant. After thresholding, the specific area within $1 \mathrm{~mm}$ from the implant surface was reconstructed and defined as the volume of interest (VOI) (Xu et al., 2018), the percentage of bone volume (BV) to the total tissue volume (TV; $\mathrm{BV} / \mathrm{TV} \%$ ) within the VOI was calculated, trabecular thickness (TbTh; $\mathrm{mm}$ ), trabecular number $(\mathrm{TbN} ; 1 / \mathrm{mm})$, and trabecular spacing $(\mathrm{TbSp} ; \mathrm{mm})$ were calculated by using its auxiliary software (SCANCO VivaCT80, Switzerland) (Chai et al., 2012; Ji et al., 2020).

\section{Histological Observations}

After micro-CT analysis, the harvested samples $(n=3)$ at each time point and groups were dehydrated through a series of increasing ethanol concentrations (70 100\%) and subsequently embedded in methyl methacrylate. The embedded samples were then longitudinally cut into $20 \mu \mathrm{m}$ sections using a diamond saw (Leica SP-1600, Germany) and stained with $1 \%$ methylene blue (Sigma) and $0.3 \%$ basic fuchsin (Sigma) solutions for qualitative and quantitative histological observations.

To obtain the histological overview of each bone-implant interface and perform histomorphometry, the histological slides were scanned (Dimage Scan Elite 5400II, Konica Minolta Photo Imaging Inc., Tokyo, Japan). Subsequently, the acquired images were subject to the quantitative analysis for new bone using Adobe Photoshop CS5 software regarding the bone-implant contact (BIC) and bone area (BA). The bone-implant gap was defined as the interface area. The new bone growth at the interface were analyzed. The bone-implant contact defined as the percentage of the implant perimeter in direct contact with the mineralized bone tissue. The bone area was calculated as the area percentage of new bone in the interface area. Besides, the newly formed bone at the bone-implant interface was observed by observing the slides at a light 

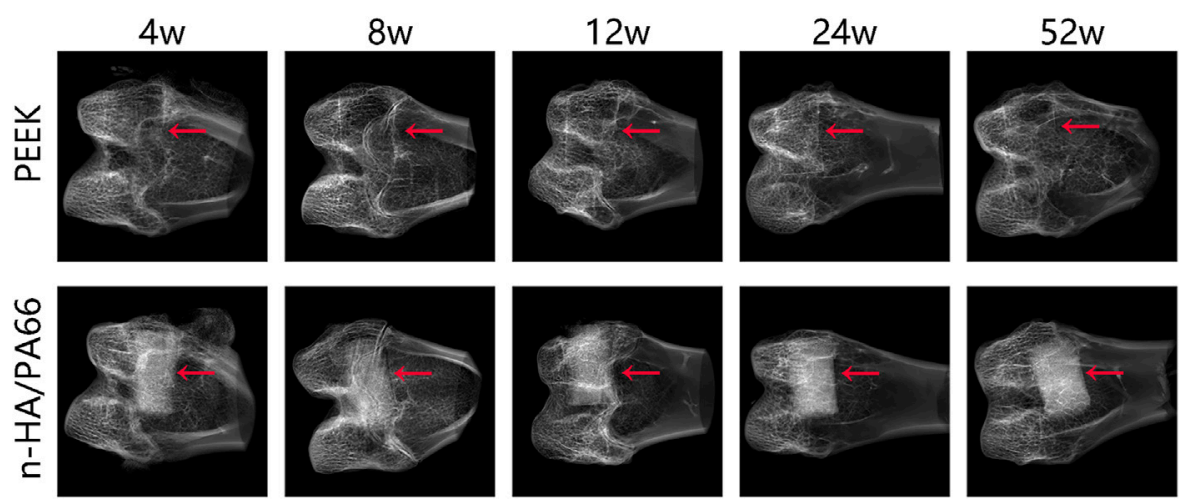

FIGURE 2 |X-ray radiographs of the bone-implant interface with polyether-ether-ketone (PEEK) and nanohydroxyapatite/polyamide 66 (n-HA/PA66) implants at 4, 8, 12, 24, and 52 weeks. (red arrow: interface).

microscope under high magnification (Nikon Eclipse E200, Tokyo, Japan) (Peng et al., 2020).

\section{SEM and EDX Analysis}

The newly retrieved specimens $(n=3)$ were cut longitudinally into $1 \mathrm{~mm}$ thick sections using a microtome (Leica SP-1600). The sections were then coated with a thin layer of gold, and the microstructure of the bone-implant interfaces was observed via SEM (ThermoFisher, Apreo S, $15 \mathrm{kV}$ ), and elemental analyses were subsequently conducted using EDX (ThermoFisher, Aztec $\mathrm{X}$-Max80) and elemental mapping to determine the interface composition at each time point $(n=3)$.

\section{Push-Out Tests}

To investigate the integrated strength of the bone-implant interface, push-out tests were performed using an electric universal testing apparatus (SHIMADZU, AGS-X-10 KN) at 24 and 52 weeks. The newly harvested bone specimens from each group were stored at $-80^{\circ} \mathrm{C}$, and were measured within $24 \mathrm{~h}$ after sacrificing the animals $(n=3)$. Peri-implant soft tissue was carefully removed and exposure the outside of the implant. The specimens were fixed into the self-curing resin for $30 \mathrm{~min}$ for making the long axis of the implant consistent with the direction of the applied force. The tests were performed at a loading rate of $5 \mathrm{~mm} / \mathrm{min}$ until the bone and implant interface was destroyed (Figures 6A,B). The load-displacement curve was obtained and the pressure at the point of a sudden drop in the push-out stress of the specimen was recorded (Xiu et al., 2016; Wang et al., 2019).

\section{STATISTICS}

Data are presented as mean \pm standard deviation (SD). Statistical analyses were performed using SPSS16.0 software, and analyzed using analysis of variance (ANOVA) followed by Fisher's multiple comparison tests. $p<0.05$ was considered statistically significant.

\section{RESULTS}

\section{X-ray Observations}

According to the X-ray radiographs (Figure 2), the n-HA/PA66 implant was conducive to observation and evaluation at the boneimplant interface because of its high density. The appearance of the defect after implantation could be clearly seen in the PEEK group because of the radiolucent nature of PEEK. After implantation for 4 weeks, no lucency or gap could be identified between the bone and implant at either of the interfaces. However, after implantation for 8 weeks, we found that PEEK implants began to exhibit a peek-halo effect, and a similar radiolucent gap formed around the n-HA/PA66 implant. In the PEEK group, the peek-halo effect continued to be identified around PEEK implants and did not seem to change during the healing process at 12,24 , and 52 weeks. In the n-HA/PA66 group, we discovered that the radiolucent gap was considerable at 8 weeks but began to narrow and decrease gradually from weeks 24 to 52 , which is consistent with the healing process.

\section{Micro-CT Analysis}

New bone formation around the implants was evaluated at 4,8 , 12,24 , and 52 weeks postoperatively using micro-CT. The crosssectional and 3D images of all implants are shown in Figures 3A,B. The implants (yellow) and newly formed bone tissue (red) in identically sized ROIs were reconstructed using $3 \mathrm{D}$ reconstructed images. Further quantitative analysis of the newly formed bone around the implants is shown in Figure 3C-F. At 4, 8, and 12 weeks after implantation, sparse new bone tissue growth at the interface (or gap) could be clearly observed. More newly formed bone was observed around the n-HA/PA66 than PEEK implants. With the prolongation of the implanting time, as part of the healing process, the new bone tissue gradually transformed into a more mature trabecular bone or woven bone at the later stage ( 24 and 52 weeks).

According to the quantitative analysis, at $4,8,12$, and 52 weeks after implantation, n-HA/PA66 implants had higher 


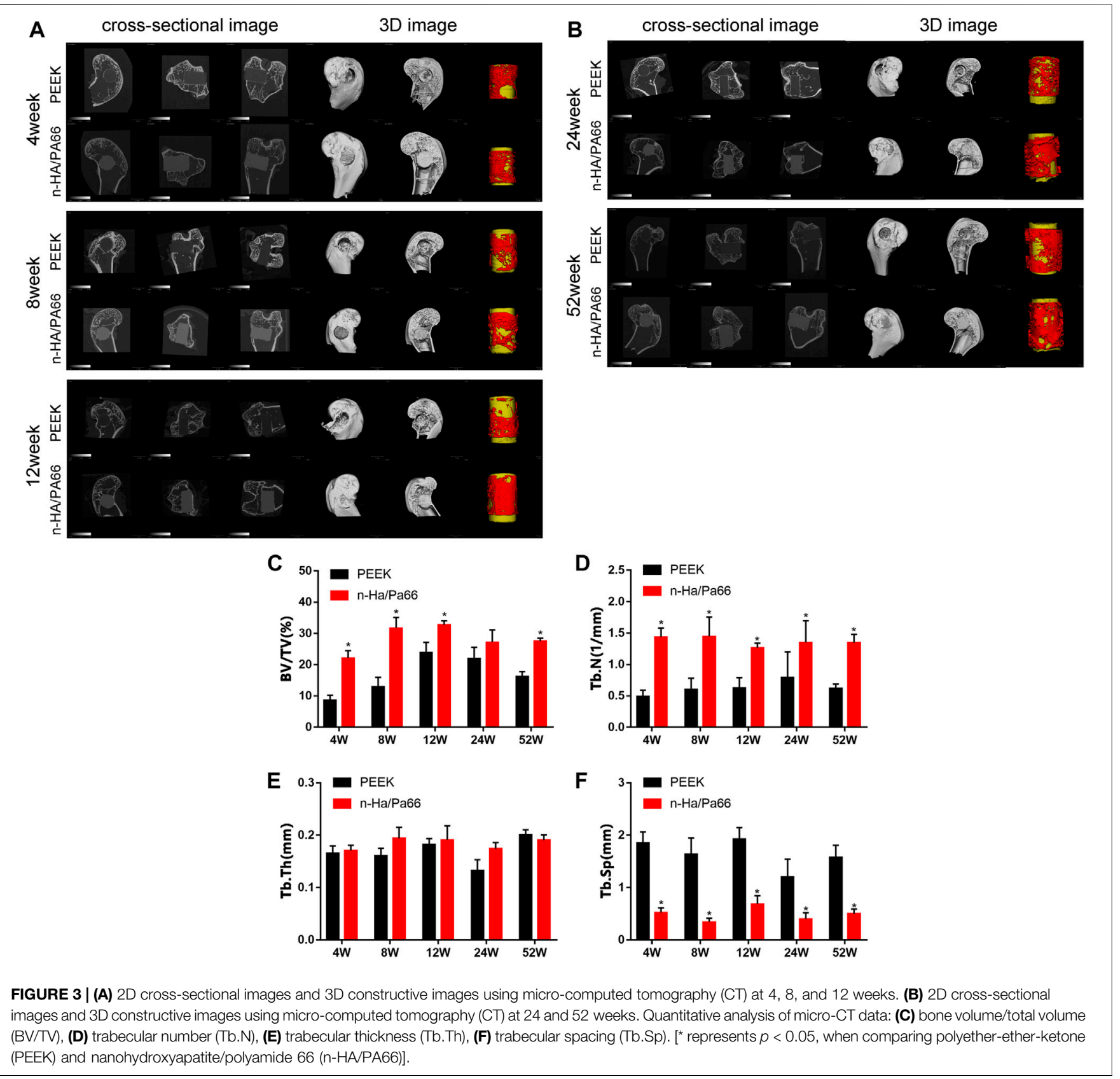

$\mathrm{BV} / \mathrm{TV}$ and $\mathrm{TbN}$ indices than the PEEK implants indicating greater new bone formation $(p<0.05, n=3)$. At all time points after implantation, PEEK implants had significantly higher TbSp values $(p<0.05, n=3)$. This indicates that the trabecular bone around the PEEK was sparse. These results proved that the quality of bone tissue around n-HA/PA66 was better than that of PEEK.

\section{Histological Observations}

After micro-CT analysis, all the gross specimens showed direct contact with the surrounding bones. Fuchsin and methylene blue staining shows the tissue response to the
PEEK and n-HA/P66 implants after 4, 8, 12, 24, and 52 weeks (Figures 4A,B). Further quantitative analysis of the new bone area rate $(\mathrm{BA} \%)$ and bone-implant contact ratio (BIC) are shown in Figures 4C,D. No special inflammatory reaction was observed in any region of the implant during the entire implantation period.

For the n-HA/PA66 implants, in the early weeks after implantation from 4 to 12 weeks, the results showed there was a "interface" between the bone and implant. Many immature woven bones filled the interface around the implant and gradually grew on the surface of the n-HA/ PA66. Moreover, with the prolongation of the implantation 


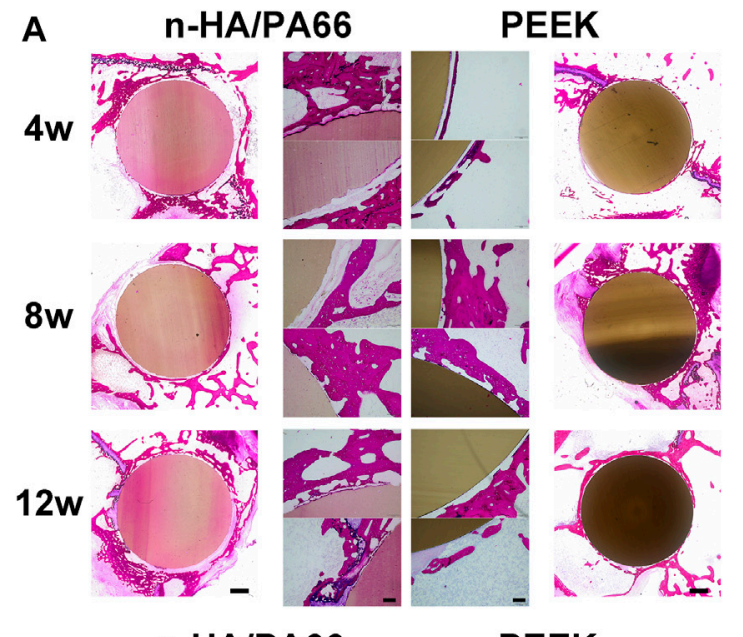

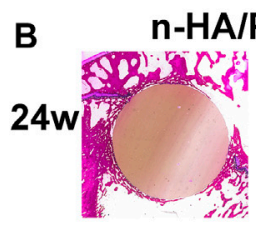
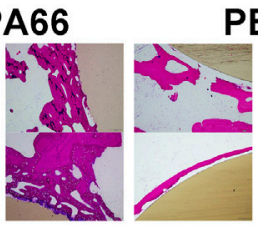

PEEK
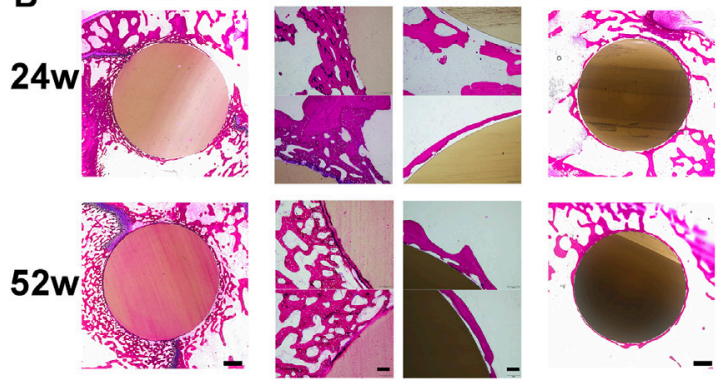
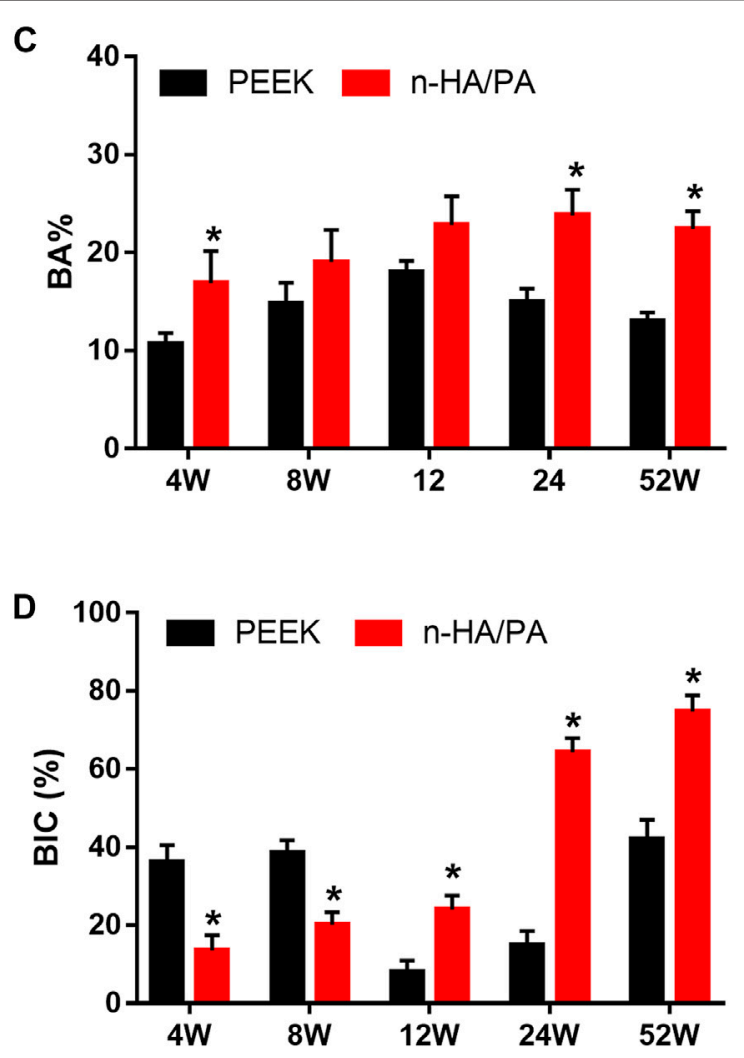

FIGURE 4 | (A) Histological overviews of polyether-ether-ketone (PEEK; left) and nanohydroxyapatite/polyamide 66 (n-HA/PA66; right) bone-implant interfaces at 4,8 , and 12 weeks (scale = $2 \mathrm{~mm}$ ); Middle: detailed light microscopic images of the selected region in the histological overviews (scale = $250 \mathrm{~mm}$ ); methylene blue and basic fuchsin staining. (B) Histological overviews of PEEK- and n-HA/PA66 bone-implant interfaces at 24 and 52 weeks (scale = 2 mm). (C) The new bone area rate (BA $\%)$ and (D) bone-implant contact ratio (BIC) were analyzed from the images shown in $\mathrm{A}$ and $\mathrm{B}$. $\left({ }^{\star} p<0.05\right)$.

time, the immature woven bone gradually disappeared, accompanied by growth of new trabecular bone around the implant surface. At 24 and 52 weeks after implantation, the formed trabecular bone on the n-HA/PA66 implants was continuous and bonded tightly to the implant surface, and excellent osseointegration could be confirmed by the images at the interface area (Figure 4B). In the PEEK group, we could easily identify the poor osseointegrated interface (or gap) on histological sections at all time points, which could possibly be missed on the X-ray. With time, the layer of fibrous encapsulation became thinner than before; however, it persisted. Furthermore, almost no visible bone tissue formation was observed, and only a small number of new bones were sparsely distributed around the PEEK implants.

\section{SEM and Surface Element Analysis}

Typical SEM images of the bone-implant sections and their elemental analyses are shown in Figures 5A,B. The new bones bonded to the n-HA/PA66 surface directly, and the gap between the new bone and the implant narrowed as the implantation time increased. In the PEEK group, the gap between the bone and implant could still be observed at each time point. At 24 and 52 weeks after implantation, excellent osteointegration between the host bone and the implant could be confirmed by the local magnified images at the interface area, and a continuity zone of n-HA/PA66 adhered to the bone without intervening space could be seen clearly (Figure 5B).

Electron microscopy of the bone-implant interface revealed interesting ultrastructural information, but it is difficult to identify the type of tissue around the implant. The EDX analysis of newly formed bone at the bone-implant interface revealed that the n-HA/PA66-bone interface contained $\mathrm{Ca}$ and $\mathrm{P}$ at a $\mathrm{Ca} / \mathrm{P}$ ratio of approximately 1.59 . The PEEK-bone interface showed less calcification and a lower $\mathrm{Ca} / \mathrm{P}$ ratio (Table 1 ).

\section{Biomechanical Push-Out Test}

Push-out tests were conducted on the specimens after implantation for 24 and 52 weeks (Figure 6A). The typical load-displacement curves, along with the average maximum push-out force (Fmax) of the specimens are shown in Figure 6B. For all the specimens, the loading force increased gradually with increasing displacement until the bone-implant interface was destroyed, indicating the Fmax. PEEK had a low bonding strength with the surrounding bone; the Fmax were $272.6 \pm 5.5$ at 24 weeks and $320.3 \pm 13.7 \mathrm{~N}$ at 52 weeks. However, the Fmax of n-HA/PA66 were $518.6 \pm 9.4$ and $530.4 \pm 8.8 \mathrm{~N}$ at 24 and 52 weeks, respectively. Accordingly, the maximum failure load of n-HA/PA66 implants was greater than that of PEEK implants at $24(18.3 \pm 0.11$ vs. $9.6 \pm 0.10 \mathrm{MPa} ; p<$ 

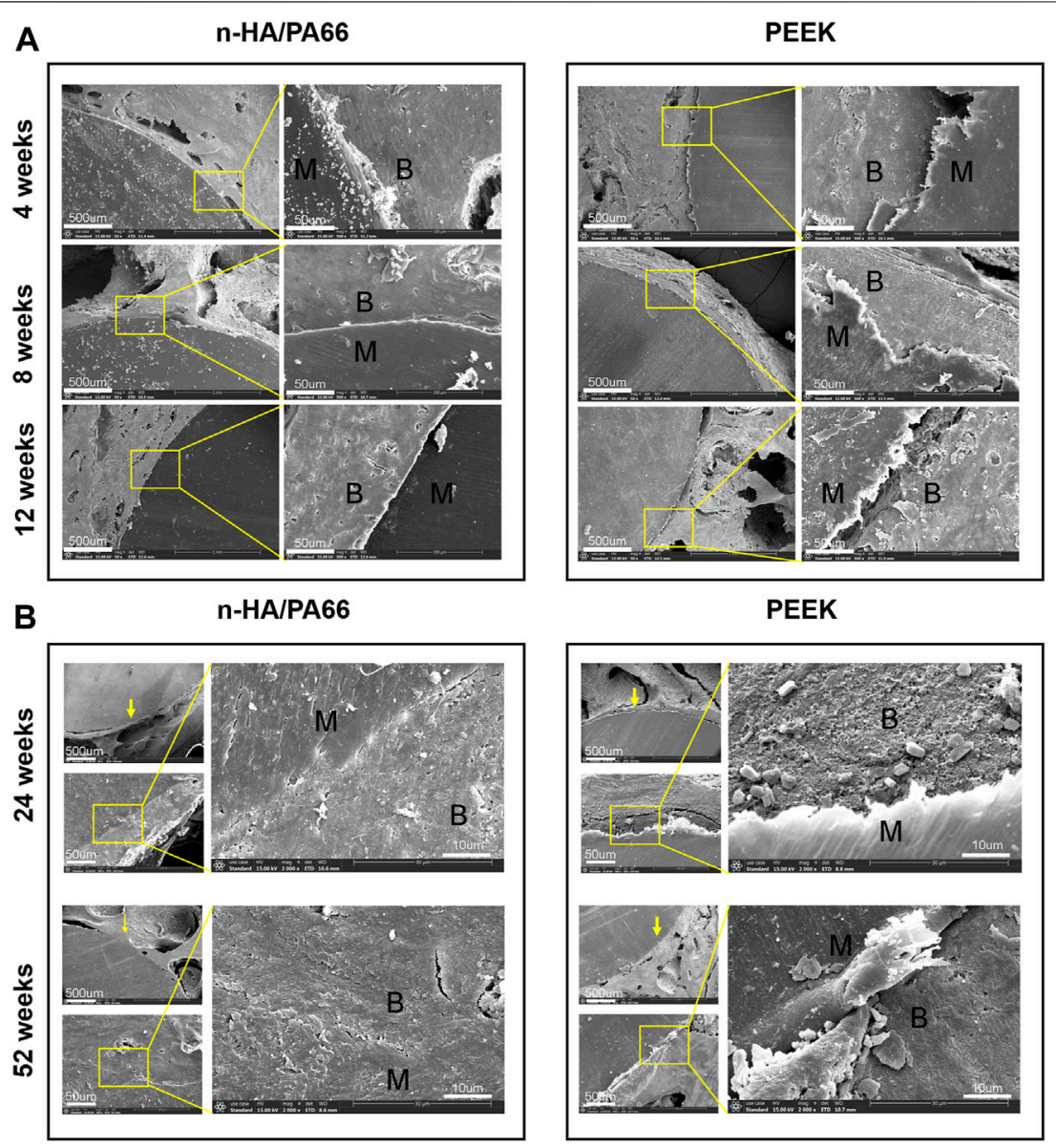

FIGURE 5 | (A) Scanning electron microscopy (SEM) image of the bone-implant interface 4, 8, and 12 weeks after implantation. (B) SEM image of the bone-implant interface 24 and 52 weeks after implantation.

\begin{tabular}{|c|c|c|c|c|c|}
\hline Sample & 4 weeks & 8 weeks & 12 weeks & 24 weeks & 52 weeks \\
\hline n-HA/PA66 & $1.47 \pm 0.03$ & $1.53 \pm 0.06$ & $1.52 \pm 0.11$ & $1.57 \pm 0.04^{\mathrm{a}}$ & $1.59 \pm 0.05^{a}$ \\
\hline
\end{tabular}

${ }^{a} p<0.05$, versus PEEK.

$0.05)$ and 52 weeks $(18.6 \pm 0.18$ vs. $11.7 \pm 0.09 \mathrm{MPa} ; p<0.05)$. Thus, the integrated strength of n-HA/PA66 was significantly higher than that of PEEK (Figures 6C, D).

\section{DISCUSSION}

Although radiolucent zones around the implant may be an indication that the implant is encapsulated by fibrous tissue, the lack of such zones does not indicate osseointegration (Albrektsson and Jacobsson, 1987). The poor osseointegration of PEEK is often attributed to its relatively bio-inert and hydrophobic properties. In contrast, n-HA/PA66 is a bioactive composite made by infiltrating nano-HA into PA66; it mimics natural bone in that the apatite is distributed within a collagen matrix (Xu et al., 2018). Thus, the composite possesses both the bioactive effects of HA and the mechanical strength of PA66. We hypothesized that the bioactive n-HA/PA66 would enhance the in vivo osseointegration effects at the bone-implant interface. We explored whether the "radiolucent gap" and "Peek-halo" were similar and if an improved bone-implant interface was exhibited with n-HA/PA66 in comparison to that with PEEK. To determine the difference between the radiolucent gaps of the two implants between the bone and the implant we assessed the differences in 
A
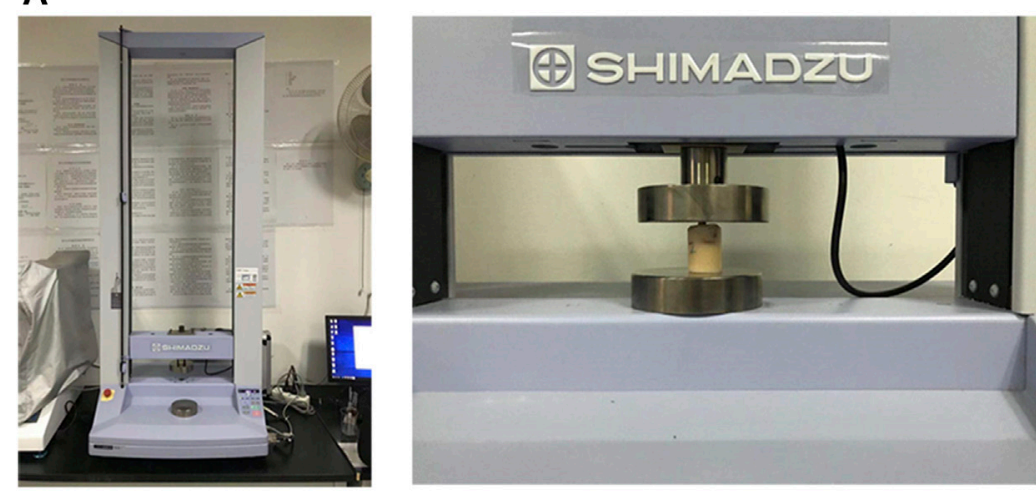

B

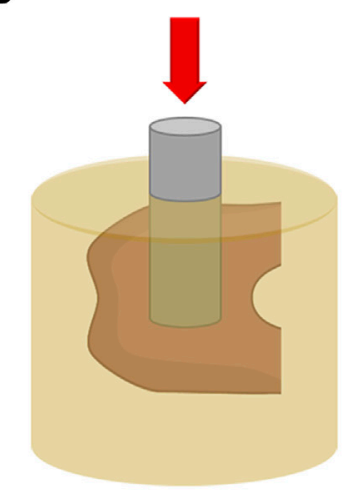

C

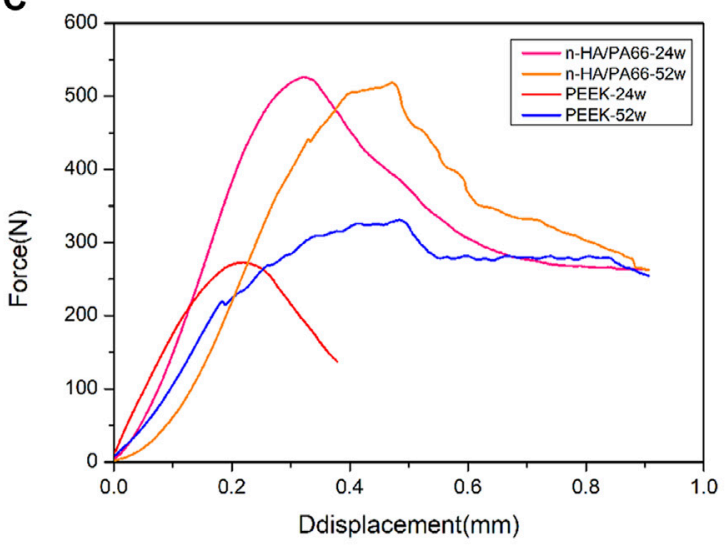

D

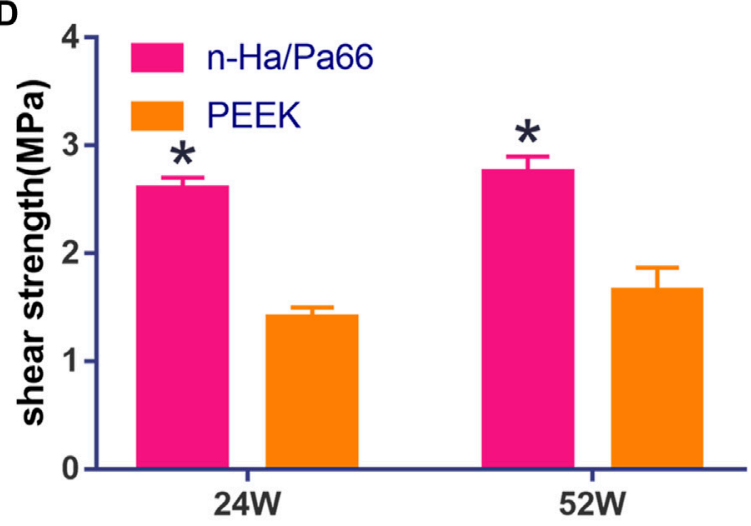

Time

FIGURE 6 | (A,B): Schematic image shows the design of the pushing-out test. (C) Typical push-out force displacement curve of the polyether-ether-ketone (PEEK) and nanohydroxyapatite/polyamide 66 (n-HA/PA66) bone-implant interface. (D) Average shear strength of the two implants at 24 and 52 weeks. ( $\left.{ }^{\star} p<0.05\right)$.

osseointegration effects of the two implants after implantation for $4,8,12,24$, and 52 weeks. These results demonstrate that the n-HA/PA66 implant exhibited a better osseointegrated boneimplant interface than PEEK. We found a radiolucent line around n-HA/PA66 in the early weeks after implantation, and the zone decreased and disappeared gradually. The PEEK implant showed a fibrous inert interface and less bone formation. The PEEK-halo line could be seen clearly during long-term observation. In addition, the push-out tests also demonstrated that the interface of n-HA/PA66 had a higher integrated strength than the PEEK implant.

Osseointegration has been used to describe the successful healing of an implant within the host bone for a long time (Kuzyk and Schemitsch, 2011; Shah et al., 2019). Knowledge of this bone-implant interface is useful for interpreting the reasoning behind orthopedic implant design strategies and their long-term success, and it is imperative for surgeons to have a basic understanding of the bone-implant interface healing process (Shah et al., 2019). In the present study, we discovered a radiolucent gap at the margin of the n-HA/PA66 implant by X-ray radiography and histological sections in the early weeks after implantation ( $4-8$ weeks). Subsequently, the gap decreased and disappeared gradually at 24 weeks and becoming more narrow at 52 weeks, which is consistent with the healing process. Through histological analysis, we could clearly observe the space between the bone and implant in the early weeks after implantation (4-12 weeks). The space was gradually filled with new bone, which suggested that n-HA/PA66 had good osseointegration properties with the surrounding bones. At 24 and 52 weeks, we found that n-HA/PA66 could integrate and be connected with host bone in some regions where new bone grew into the n-HA/PA66 implant after implantation. In contrast, in the PEEK group, a radiolucent halo line could be identified by $\mathrm{X}$-ray film at 4 and 8 weeks, and the fibrous gap could be clearly observed by histological assessment. Furthermore, there was no change in the fibrous interface during the healing process after 12, 24, and 52 weeks. This indicates that the PEEK-bone interface becomes inert, and the surrounding tissue has formed a stable bioinert structure. The same results were reported by Walsh and etc. who tried to incorporate HA into PEEK and plasma spraying of titanium to PEEK in order to improve osseointegration (Pelletier et al., 2016; Walsh et al., 2018). 
Clinically, the success or failure of an implanted device is often measured by X-ray radiography and CT examination. The "PEEK-halo" and "radiolucent gap" effects in many clinical studies reflect the inadequacy of clinical methods for evaluating osseointegration. These tests are indirect methods, that indicate, but do not verify osseointegration. The classical description of osseointegration is a direct contact between the implant surface and bone at the light microscopic level (Shah et al., 2019), which is mainly distinguished from fibrous integration. However, this definition ignores the presence of other interposed components of the tissue along the interface. The interface should include bone-implant areas and the periimplant bone. Micro-CT is frequently used to evaluate bone formation around the implant, but the bone-implant interface is often obscured and cannot be clearly determined (Palmquist et al., 2017). In the present study, the results of micro-CT and histological analysis showed that the n-HA/PA66 implant had higher BV/TV values and bone volumes than PEEK at 4,8 , and 12 weeks, which remained stable from 24 to 52 weeks. This demonstrated that the n-HA/PA66 surface may favor new bone formation. To assert that an implant achieved good osseointegration and solid fusion must imply that most of it is anchored with bone tissue. We therefore investigated the ultrastructure of the bone-implant interface using SEM and EDX mapping. Electron microscopy of the tissue interface is capable of producing interesting information, but it is difficult to identify the precise components of tissue around the entire implant. The results demonstrated that the n-HA/PA66 implant has a thin interface gap and a continuous mineralized interface zone, and the EDX analysis showed that a fiber medial layer was found between the PEEK implant and bone, whereas the n-HA/PA66 implant exhibited an improved mineralized implant-bone interface.

The aim of determining an optimized spinal fusion device is to achieve good osseointegration and promote long-term success (Shah et al., 2019). Through push-out tests, we could see that n-HA/PA66 implants can possess stronger bonding strength with the surrounding bone tissue and could obtain sufficient stability at 24 and 52 weeks, whereas the PEEK implant could be pushed out easily, which indicates that the surrounding bone tissue cannot adhere to PEEK. Therefore, our hypothesis that n-HA/ PA66 would have better osseointegration properties and higher bonding strength than PEEK was confirmed. Several efforts have been made to improve the bioactivity and osteointegration properties of PEEK to prolong its long-term stability (Johansson et al., 2015; Torstrick et al., 2017; Liu et al., 2018); for example, plasma-sprayed titanium or HA coatings on PEEK and the incorporation of HA into PEEK (Walsh et al., 2016; Torstrick et al., 2017). However, these studies only showed good improvements in bone formation by histological analysis and micro-CT evaluation. Bone formation begins at the implant surface in response to the surface physicochemical properties of the implant surface (Liu et al., 2018). Remodeling of the bone in contact with the implant surface continues throughout the lifetime of the implant. This remodeling may allow for increased contact between the implant and the bone over time and likely continues for longer than six months. Our previous studies have shown that a bone-like apatite layer can be formed on the surface of n-HA/PA66 in simulated body fluid (Wang et al., 2002), and the n-HA/PA66 composite could promote the growth and osteogenic differentiation of mesenchymal stem cells (Xu et al., 2018). The EDX mapping results revealed that $\mathrm{Ca}$ and phosphonium were successfully incorporated on the surface of n-HA/PA66 and were homogeneously distributed. Thus, the n-HA particles on the surface provide a good osteoconductive surface, which has a positive effect on bone apposition and mineralization.

In summary, we found that the n-HA/PA66 implant has good osseointegration properties and exhibits an improved boneimplant interface. Thus, this composite is appropriate for bone formation and bonding with the surrounding bone and provides better biomechanical stability than PEEK. Consequently, the n-HA/PA66 composite exhibited good osseointegration properties for clinical applications. Additional clinical and experimental studies are required to elucidate the precise bonding mechanisms underlying the bone-implant interface.

\section{DATA AVAILABILITY STATEMENT}

The raw data supporting the conclusions of this article will be made available by the authors, without undue reservation.

\section{ETHICS STATEMENT}

The animal study was reviewed and approved by The Ethical Committee of The First Affiliated Hospital of Chongqing Medical University.

\section{AUTHOR CONTRIBUTIONS}

JL and HP performed the experiments, analyzed and interpreted the results. $\mathrm{HP}, \mathrm{CH}, \mathrm{ZC}$, and $\mathrm{TH}$ performed the animal experiments. JL, HL, and ZQ acquired the research operating funding, advised on the conception and conduction of the experiments and data analysis.

\section{FUNDING}

The authors thank Science and Health Project of Chongqing Health Commission(2021MSXM143) and Research and Innovation Project of Graduate students in Chongqing (CYB19142) for providing funding for this research. 


\section{REFERENCES}

Albrektsson, T., and Jacobsson, M. (1987). Bone-metal Interface in Osseointegration. The J. Prosthetic Dentistry 57 (5), 597-607. doi:10.1016/ 0022-3913(87)90344-1

Bagby, G. W. (1988). Arthrodesis by the Distraction-Compression Method Using a Stainless Steel Implant. Orthopedics 11 (6), 931-934. doi:10.3928/0147-744719880601-13

Bertollo, N., Matsubara, M., Shinoda, T., Chen, D., Kumar, M., and Walsh, W. R. (2011). Effect of Surgical Fit on Integration of Cancellous Bone and Implant Cortical Bone Shear Strength for a Porous Titanium. The J. Arthroplasty 26 (7), 1000-1007. doi:10.1016/j.arth.2010.12.007

Chai, Y. C., Kerckhofs, G., Roberts, S. J., Van Bael, S., Schepers, E., Vleugels, J., et al. (2012). Ectopic Bone Formation by 3D Porous Calcium Phosphate-Ti6Al4V Hybrids Produced by Perfusion Electrodeposition. Biomaterials 33 (16), 4044-4058. doi:10.1016/j.biomaterials.2012.02.026

Cloward, R. B. (2007). The Anterior Approach for Removal of Ruptured Cervical Disks. J. Neurosurg. Spine. 6 (5), 496-511. doi:10.3171/spi.2007.6.5.496

Ji, M., Chen, H., Yan, Y., Ding, Z., Ren, H., and Zhong, Y. (2020). Effects of Tricalcium Silicate/sodium Alginate/calcium Sulfate Hemihydrate Composite Cements on Osteogenic Performances In Vitro and In Vivo. J. Biomater. Appl. 34 (10), 1422-1436. doi:10.1177/0885328220907784

Johansson, P., Jimbo, R., Kozai, Y., Sakurai, T., Kjellin, P., Currie, F., et al. (2015). Nanosized Hydroxyapatite Coating on PEEK Implants Enhances Early Bone Formation: A Histological and Three-Dimensional Investigation in Rabbit Bone. Materials 8 (7), 3815-3830. doi:10.3390/ma8073815

Kurtz, S. M., and Devine, J. N. (2007). PEEK Biomaterials in Trauma, Orthopedic, and Spinal Implants. Biomaterials 28 (32), 4845-4869. doi:10.1016/j. biomaterials.2007.07.013

Kuzyk, P., and Schemitsch, E. (2011). The Basic Science of Peri-Implant Bone Healing. Indian J. Orthop. 45 (2), 108-115. doi:10.4103/0019-5413.77129

Liu, W., Li, J., Cheng, M., Wang, Q., Yeung, K. W. K., Chu, P. K., et al. (2018). ZincModified Sulfonated Polyetheretherketone Surface with Immunomodulatory Function for Guiding Cell Fate and Bone Regeneration. Adv. Sci. 5 (10), 1800749. doi:10.1002/advs.201800749

Noiset, O., Schneider, Y.-J., and Marchand-Brynaert, J. (1999). Fibronectin Adsorption Or/and Covalent Grafting on Chemically Modified PEEK Film Surfaces. J. Biomater. Sci. Polym. Edition 10 (6), 657-677. doi:10.1163/ 156856299x00865

Palmquist, A., Shah, F. A., Emanuelsson, L., Omar, O., and Suska, F. (2017). A Technique for Evaluating Bone Ingrowth into 3D Printed, Porous Ti6Al4V Implants Accurately Using X-ray Micro-computed Tomography and Histomorphometry. Micron 94, 1-8. doi:10.1016/j.micron.2016.11.009

Pelletier, M. H., Cordaro, N., Punjabi, V. M., Waites, M., Lau, A., and Walsh, W. R. (2016). PEEK versus Ti Interbody Fusion Devices. Clin. Spine Surg. 29 (4), E208-E214. doi:10.1097/BSD.0b013e31826851a4

Peng, H., Li, J., Xu, Y., and Lv, G. (2020). Icaritin Enhancing Bone Formation Initiated by Sub-microstructured Calcium Phosphate Ceramic for Critical Size Defect Repair. Front. Mater. 7, 598057. doi: $10.3389 /$ fmats.2020.598057

Phan, K., Hogan, J. A., Assem, Y., and Mobbs, R. J. (2016). PEEK-halo Effect in Interbody Fusion. J. Clin. Neurosci. 24, 138-140. doi:10.1016/j.jocn.2015.07.017

Shah, F. A., Thomsen, P., and Palmquist, A. (2019). Osseointegration and Current Interpretations of the Bone-Implant Interface. Acta Biomater. 84, 1-15. doi:10. 1016/j.actbio.2018.11.018

Torstrick, F. B., Lin, A. S. P., Potter, D., Safranski, D. L., Sulchek, T. A., Gall, K., et al. (2018). Porous PEEK Improves the Bone-Implant Interface Compared to Plasma-Sprayed Titanium Coating on PEEK. Biomaterials 185, 106-116. doi:10.1016/j.biomaterials.2018.09.009
Torstrick, F. B., Safranski, D. L., Burkus, J. K., Chappuis, J. L., Lee, C. S. D. Guldberg, R. E., et al. (2017). Getting PEEK to Stick to Bone: The Development of Porous PEEK for Interbody Fusion Devices. Tech. Orthop. 32 (3), 158-166. doi: $10.1097 /$ bto. 0000000000000242

Toth, J. M., Wang, M., Estes, B. T., Scifert, J. L., Seim, H. B., 3rd, and Turner, A. S. (2006). Polyetheretherketone as a Biomaterial for Spinal Applications. Biomaterials 27 (3), 324-334. doi:10.1016/j.biomaterials.2005.07.011

Walsh, W. R., Bertollo, N., Christou, C., Schaffner, D., and Mobbs, R. J. (2015). Plasma-sprayed Titanium Coating to Polyetheretherketone Improves the Bone-Implant Interface. Spine J. 15 (5), 1041-1049. doi:10.1016/j.spinee.2014.12.018

Walsh, W. R., Pelletier, M. H., Bertollo, N., Christou, C., and Tan, C. (2016). Does PEEK/HA Enhance Bone Formation Compared with PEEK in a Sheep Cervical Fusion Model? Clin. Orthop. Relat. Res. 474 (11), 2364-2372. doi:10.1007/ s11999-016-4994-x

Walsh, W. R., Pelletier, M. H., Christou, C., He, J., Vizesi, F., and Boden, S. D. (2018). The In Vivo Response to a Novel Ti Coating Compared with Polyether Ether Ketone: Evaluation of the Periphery and Inner Surfaces of an Implant. Spine J. 18 (7), 1231-1240. doi:10.1016/j.spinee.2018.02.017

Wang, H., Su, K., Su, L., Liang, P., Ji, P., and Wang, C. (2019). Comparison of 3D-Printed Porous Tantalum and Titanium Scaffolds on Osteointegration and Osteogenesis. Mater. Sci. Eng. C 104, 109908. doi:10.1016/j.msec. 2019.109908

Wang, X., Li, Y., Wei, J., and de Groot, K. (2002). Development of Biomimetic Nano-Hydroxyapatite/poly(hexamethylene Adipamide) Composites. Biomaterials 23 (24), 4787-4791. doi:10.1016/s0142-9612(02)00229-6

Wu, S.-H., Li, Y., Zhang, Y.-Q., Li, X.-K., Yuan, C.-F., Hao, Y.-L., et al. (2013). Porous Titanium-6 Aluminum-4 Vanadium Cage Has Better Osseointegration and Less Micromotion Than a Poly-Ether-Ether-Ketone Cage in Sheep Vertebral Fusion. Artif. Organs 37 (12), E191-E201. doi:10. 1111/aor.12153

Xiu, P., Jia, Z., Lv, J., Yin, C., Cheng, Y., Zhang, K., et al. (2016). Tailored Surface Treatment of 3D Printed Porous Ti6Al4V by Microarc Oxidation for Enhanced Osseointegration via Optimized Bone In-Growth Patterns and Interlocked Bone/Implant Interface. ACS Appl. Mater. Inter. 8 (28), 17964-17975. doi:10. 1021/acsami.6b05893

Xu, Y., Li, H., Wu, J., Yang, Q., Jiang, D., and Qiao, B. (2018). Polydopamineinduced Hydroxyapatite Coating Facilitates Hydroxyapatite/polyamide 66 Implant Osteogenesis: an In Vitro and In Vivo Evaluation. Int. J. Nanomedicine. Vol. 13, 8179-8193. doi:10.2147/ijn.S181137

Yuan, B., Cheng, Q., Zhao, R., Zhu, X., Yang, X., Yang, X., et al. (2018). Comparison of Osteointegration Property between PEKK and PEEK: Effects of Surface Structure and Chemistry. Biomaterials 170, 116-126. doi:10.1016/j. biomaterials.2018.04.014

Zhang, Y., Deng, X., Jiang, D., Luo, X., Tang, K., Zhao, Z., et al. (2016). Long-term Results of Anterior Cervical Corpectomy and Fusion with NanoHydroxyapatite/polyamide 66 Strut for Cervical Spondylotic Myelopathy. Sci. Rep. 6, 26751. doi:10.1038/srep26751

Conflict of Interest: The authors declare that the research was conducted in the absence of any commercial or financial relationships that could be construed as a potential conflict of interest.

Copyright $\odot 2021 \mathrm{Li}$, Peng, Chen, $\mathrm{Hu}, \mathrm{He}$, Li and Quan. This is an open-access article distributed under the terms of the Creative Commons Attribution License (CC BY). The use, distribution or reproduction in other forums is permitted, provided the original author(s) and the copyright owner(s) are credited and that the original publication in this journal is cited, in accordance with accepted academic practice. No use, distribution or reproduction is permitted which does not comply with these terms. 